\title{
Hyperspectral imaging of retinal microvascular anatomy
}

\author{
Amir H. Kashani ${ }^{1}$, Mark Wong ${ }^{2}$, Nicole Koulisis ${ }^{1,}{ }^{3}$, Chein-I Chang ${ }^{4}$, Gabriel Martin ${ }^{5}$, Mark S. \\ Humayun ${ }^{1,6,7}$
}

1. USC Eye Institute, Department of Ophthalmology, Keck School of Medicine of the University of Southern California, Los Angeles, CA, USA. 2. National Aeronautics and Space Administration, Washington, DC, USA. 3. University of Massachusetts Medical School, Worcester, MA, USA. 4. Department of Computer Science and Electrical Engineering, University of Maryland, Baltimore County, Baltimore, MD, USA. 5. Reichert Technologies, Depew, NY, USA. 6. Department of Biomedical Engineering, Viterbi School of Engineering, University of Southern California, Los Angeles, CA, USA. 7. Department of Cell \& Neurobiology, Keck School of Medicine of the University of Southern California, Los Angeles, CA, USA.

Correspondence: Amir H. Kashani, MD, PhD. Address: USC Eye Institute, 1450 San Pablo Street, 4th floor, CA 90033, Los Angeles, USA. Email: ahkashan@med.usc.edu

Received: August 31, 2015

Accepted: October 29, 2015 Online Published: November 22, 2015

DOI : $10.5430 /$ jbei.v2n1p139

URL: http://dx.doi.org/10.5430/jbei.v2n1p139

\section{Abstract}

Background: Hyperspectral image processing has been applied to many aspects of astronomical and earth science research. Furthermore, advances in computed tomographic imaging spectroscopy and diffraction grating design have allowed biological applications for non-invasive tissue analysis. Herein, we describe a hyperspectral computed tomographic imaging spectroscope (HCTIS) that provides high spatial, spectral and temporal resolution ideal for imaging biological tissue in vivo.

Methods: We demonstrate proof-of-principle application of the HCTIS by imaging and mapping the microvascular anatomy of the retina of a model organism (rabbit) in vivo. The imaging procedure allows rapid and dense spectral sampling, is non-toxic, non-invasive, and easily adaptable to a commercially available fundus camera system.

Results: HCTIS provides highly co-registered temporal, spatial and spectral data with resolution capable of reconstructing the fine vascular tree of the rabbit retina in vivo.

Conclusions: We show that HCTIS allows for reliable and reproducible tissue classification and detection using signature discriminant analysis. Future applications of this system may provide promising diagnostic methods for diseases of many tissues.

\section{Key words}

Hyperspectral retina processing, Hyperspectral medical image processing, Spectral angle mapper, Spectral information divergence, Relative spectral discriminatory probability 


\section{Introduction}

Hyperspectral imaging systems, which are systems capable of detecting unique spectral information for identification purposes, were first pioneered for use in fields such as the earth and planetary sciences ${ }^{[1]}$. Since then, hyperspectral imaging systems have emerged in fields including ophthalmology ${ }^{[2-4]}$, oncology ${ }^{[5,6]}$, fluorescence microscopy ${ }^{[7]}$, bioinformatics ${ }^{[8]}$, vascular physiology ${ }^{[9,10]}$, archeology and art conservation ${ }^{[11]}$, and even forensic science ${ }^{[12,13]}$. Investigators are likely drawn to the technology for its non-invasive means of detecting and characterizing an array of biological and non-biological materials. Among other systems developed, the hyperspectral computed tomographic imaging spectrometer (HCTIS) was designed specifically for the non-invasive imaging and characterization of the microvasculature in vivo at high speeds and high resolution ${ }^{[14]}$.

In contrast to the HCTIS, most spectroscopy methods in the biological sciences use band sequential scanning, which limits temporal resolution and spectral range and confounds spatial registration of data. This results in a limited signal-to-noise ratio, and an increased susceptibility to noise from pigmentation, optical media, and scatter can further confound results ${ }^{[2,15,16]}$. Moreover, these methods require a priori knowledge of characteristic target spectra, which are frequently not available.

The HCTIS combines state-of-the-art two-dimensional (2D) diffraction gratings with computed tomographic algorithms to allow simultaneous $(<3 \mathrm{msec})$ acquisition of spectra over $450-750 \mathrm{~nm}$ with approximately $4 \mathrm{~nm}$ spectral resolution ${ }^{[14,16,17]}$. There are a number of advantages to this hyperspectral imager over multi-wavelength or bandsequential scanning devices. First, there are no moving parts, nor is spatial or spectral scanning required. Therefore, there is also no need for image registration due to image movement. Second, the 2D diffraction grating design allows dense sampling of the spectral data (up to 76 bands) within the $3 \mathrm{msec}$ duration of a simple snapshot (photographic) image. Third, the computed tomographic imaging algorithms reconstruct images with high spatial-spectral resolution and allow detailed correlations of image targets that are spectrally, spatially and temporally co-registered. Fourth, the simple optical design of the hyperspectral camera is adaptable to many imaging instruments that are commercially available ${ }^{[9,16]}$. Lastly, the dense hyperspectral data set allows application of hyperspectral signature discriminant analysis that is otherwise not possible ${ }^{[18]}$. Due to the nontoxic, non-invasive nature of this imaging modality, as well as its rapid image acquisition, the HCTIS can be used in vivo and has important applications in the study of anatomy, circulatory physiology, and tissue metabolism. Here, we integrate HCTIS with a standard, FDA-approved fundus camera to image the microvasculature inside the intact rabbit eye (in vivo). This proof-of-principle application shows that the HCTIS can provide highly co-registered temporal, spatial and spectral data with sufficient resolution to allow reconstruction of the fine vascular tree. We also demonstrate that we can empirically identify retinal vessels using hyperspectral signature discriminant analysis without previous knowledge of any of the tissue's spectral components. Future application of this system in research and clinical settings can provide unique and useful information.

\subsection{Hyperspectral signature discriminant analysis}

\subsubsection{Spectral angle mapper (SAM)}

A popular way of measuring the discriminatory capacity of hyperspectral signatures is the spectral angle mapper ${ }^{[19]}$. This method has been thoroughly investigated ${ }^{[20-22]}$ and is a proven method for measuring the similarity between two or more hyperspectral signatures. This measurement also provides a discriminatory measure between two points within an image and is represented as the spectral angle $(\theta)$ in radians between the target spectrum and reference spectrum:

$$
\theta=\cos ^{-1}\left(\frac{s r}{\sqrt{s s} \sqrt{r r}}\right)
$$

where $s$ is the hyperspectral reference spectrum in the library (or tissue) and $r$ is the hyperspectral signature of the target grid value (voxel) within the image data. Moreover, the spectral angle can be viewed graphically as the angular displacement of the spectral profile between two points as shown in Figure 1. 
Figure 1. Graphical representation of the SAM measurement between two hyperspectral signatures

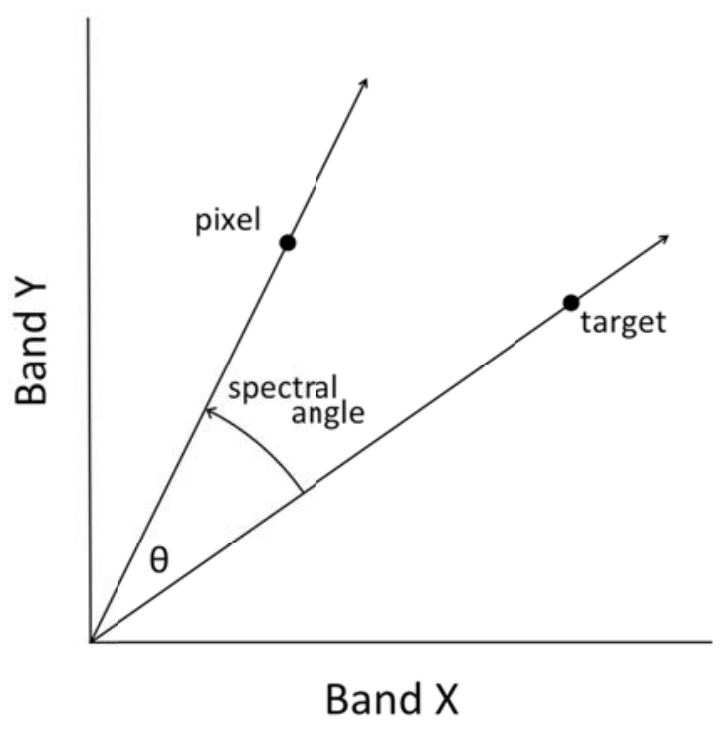

\subsubsection{Spectral information divergence (SI D)}

Another way of identifying one particular hyperspectral signature within a hyperspectral data set is called spectral information divergence ${ }^{[23]}$. This method uses the Kullback-Leibler information divergence theory and provides a better relative quantification measure than SAM. To calculate SID, the probability vectors $p$ and $q$, defined as:

$$
\begin{aligned}
& \mathrm{p}=\left(p_{1}, p_{2}, \cdots, p_{L}\right)^{r} \\
& \mathrm{q}=\left(q_{1}, q_{2}, \cdots, q_{L}\right)^{r}
\end{aligned}
$$

for the spectral signatures of two voxels $s_{p}$ and $s_{q}$, where:

$$
\begin{gathered}
p_{k}=\frac{s_{p k}}{\sum_{k=1}^{L} s_{p k}} \\
q_{k}=\frac{s_{q k}}{\sum_{k=1}^{L} s_{q k}}
\end{gathered}
$$

are assumed. The average self-information discrepancy of signature $s_{p}$ relative to $s_{q}$, noted as $D\left(s_{p} \| s_{q}\right)$, can be calculated as:

$$
D\left(s_{p} \| s_{q}\right)=\sum_{l=1}^{L} p_{l} \log \left(p_{l} / q_{l}\right)
$$

where $L$ is total number of image bands. Therefore, the SID can be calculated with the following equation:

$$
\operatorname{SID}\left(s_{p}, s_{q}\right)=D\left(s_{p} \| s_{q}\right)+D\left(s_{q} \| s_{p}\right)
$$

As shown in Equation (7), the SID is the sum of the relative self-information discrepancy of $s_{p}$ to $s_{q}$, and vice versa. It should be noted that SID is more robust than SAM under noisy conditions and produces more accurate discrepancy measurements for hyperspectral signatures ${ }^{[22]}$.

\subsubsection{Relative spectral discriminatory probability (RSDPB)}

The measurements provided by SAM or SID are a relative discriminatory analysis between one point and the rest of the image voxels. However, it does not provide a relative measure if more than one reference point is provided and does not describe which voxel has more similarity to a given reference voxel. In order to provide a likelihood measure, the 
RSDPB ${ }^{[24]}$ gives a straightforward method of calculating the probability that each voxel in a hyperspectral image is similar to a reference point in the image.

To calculate $R S D P B$, we assume $\left\{\boldsymbol{s}_{k}\right\}_{k=1}^{K}$ where $K$ is the number of spectral signatures (reference points) for a given image, otherwise referred to as the signature library $(\triangle)$. Thus, the $R S D P B$ is calculated as follows:

$$
\operatorname{RSDPB}_{t, \Delta}(k)=m\left(t, s_{k}\right) / \sum_{j=1}^{K} m\left(t, s_{j}\right)
$$

where $t$ is the target voxel in the hyperspectral image and $m\left(t, s_{k}\right)$ is the discriminatory measure between the target voxel and the reference signature. It is noted that $m\left(t, s_{k}\right)$ can be calculated with either SAM or SID.

Consequently, as the $\operatorname{RSDPB}_{t, 4}$ value becomes smaller, there is a higher likelihood of similarity to a particular reference signature. For easier graphical illustration, however, the value for $1-R S D P B_{t, 4}$ is plotted in subsequent surface plots, such that as values for $1-R S D P B_{t, 4}$ increase, so too, does the likelihood of similarity to the reference signature increase.

\section{Methods}

All animal experiments were conducted in accordance with the Institutional Animal Care and Use Committee (IACUC) at the University of Southern California. New Zealand pigmented and albino rabbits weighing 3-4 $\mathrm{kg}$ were used in the following experiments. Two sets of data were acquired from 2 different rabbits that were anesthetized and sedated with the standard intramuscular doses of xylazine and ketamine. The eyes were pharmacologically dilated for imaging. Imaging was performed using a customized hyperspectral camera (Reichert Technologies, Inc., Buffalo, NY) linked to the port of a standard Zeiss FF450 IR fundus camera. The HCTIS detailed herein has been previously reported ${ }^{[25]}$. In essence, the HCTIS acquires spatial and spectral data by imaging a scene through a 2D grating; from this image, multiple, spectrally dispersed images of the scene, in this case the retina, are projected onto a focal plane array (Retiga 2000R Camera; QImaging, Inc., BC, Canada). Prior to image processing, the dispersion pattern of the spectra onto the array is calibrated using a monochromator. All images acquired are recorded and subsequently stored on a computer using standard image acquisition software (QImaging, Inc., BC, Canada). The imaging device acquires roughly 76 spectral bands in $<3 \mathrm{msec}$ using a standard fundus flash. Thus, this rapid image acquisition offers several advantages: first, it improves signal-tonoise, as images are no longer hindered by movement from microsaccades, and second, it eliminates the need for image registration, thereby reducing motion artifact and pixel misregistration. Furthermore, since all spectral information is collected within a single snapshot, there are no moving parts in the system, and no need for time-intensive scans. All spectral information is spatially and temporally co-registered on the focal plane array. Special algorithms, based on iterative expected-maximization algorithms, are thus used to reconstruct the spectrally dispersed images into a threedimensional (3D) map of spatial (X-Y axis) and spectral (Z-axis) information that is based on individual wavelength information ${ }^{[25]}$. This $3 \mathrm{D}$ representation of spatial and spectral information is sometimes termed a "hyperspectral cube".

Two signature discriminating methods that are widely used in remote sensing, SAM and SID, were chosen to analyze the spectral characteristics of the rabbit retinal vasculature. These effective methods were originally used for endmember distinction for mineral or plants in satellite hyperspectral image data ${ }^{[26]}$. Therefore, the application of these algorithms to identify anatomic features within the retina, such as blood vessels is ideal. In addition, the RSDPB is an extension to SAM and SID and gives a probabilistic measure of similarity between any given set of signatures within the image. Experienced ophthalmologists selected six spectral reference points on each retinal image to represent either the blood vessel reference signatures or the background (nerve fiber layer) reference signatures, which are demonstrated in Figures 2 and 3. Each voxel on the hyperspectral data is then processed with SAM, SID and RSDPB using these six reference signatures. The resulting 3D plots in Figures 4 and 5 show a relative probabilistic distribution of the spectral signatures within the image based on values resulting from each calculation. 
Figure 2. Spectral reference locations in retinal image from rabbit \#1. Reference point 1 represents background. Reference point 2 represents a small vessel, and points 3-6 represent large blood vessels.

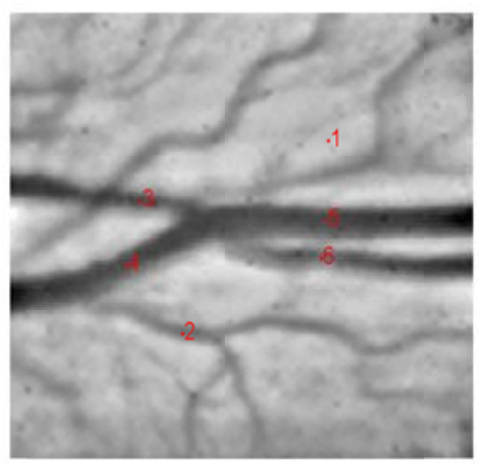

Figure 3. Spectral reference locations in retinal image from rabbit \#2. Reference points 1 and 2 represent background, and points 3-6 represent blood vessels.

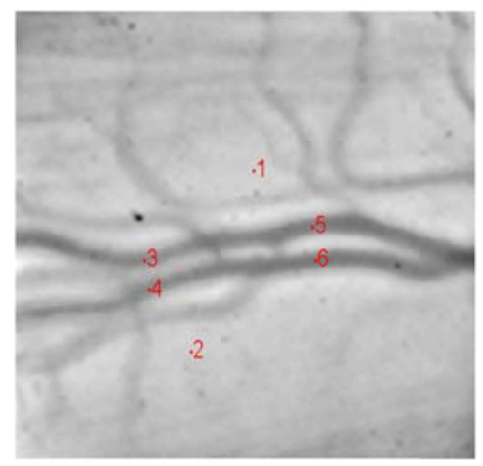

\section{Results}

Each of the spectral signature points selected in Figures 2 and 3 were then used in SAM and SID discrimination methods to measure the pixel-to-pixel signature distinction. The resulting values are further tabulated in Tables 1 and 2 . Because the order of the points does not affect the output value, the top right of Tables 1 and 2 respects the SAM results while, the bottom left shows the SID outputs. In addition, the SAM and SID results are 0 for identical spectrum; therefore, they are crossed out in the diagonal cells of the following tables.

Table 1. The SAM and SID values calculated for positions 1 to 6 as noted in Figure 2

\begin{tabular}{|c|c|c|c|c|c|c|c|}
\hline & & \multicolumn{6}{|c|}{ SAM results (Top Right) } \\
\hline & & Pos.1 & Pos.2 & Pos.3 & Pos.4 & Pos.5 & Pos.6 \\
\hline \multirow{6}{*}{$\begin{array}{l}\text { SID results } \\
\text { (Bottom } \\
\text { Left) }\end{array}$} & Pos. 1 & & $4.58 \mathrm{E}-03$ & $6.82 \mathrm{E}-03$ & $6.48 \mathrm{E}-03$ & $7.77 \mathrm{E}-03$ & $5.21 \mathrm{E}-03$ \\
\hline & Pos. 2 & $2.09 \mathrm{E}-05$ & & $2.52 \mathrm{E}-03$ & $2.40 \mathrm{E}-03$ & $3.48 \mathrm{E}-03$ & $1.72 \mathrm{E}-03$ \\
\hline & Pos. 3 & 4.64E-05 & $6.32 \mathrm{E}-06$ & & $1.30 \mathrm{E}-03$ & $1.36 \mathrm{E}-03$ & $2.00 \mathrm{E}-03$ \\
\hline & Pos. 4 & 4.19E-05 & $5.76 \mathrm{E}-06$ & $1.69 \mathrm{E}-06$ & & $2.02 \mathrm{E}-03$ & 2.04E-03 \\
\hline & Pos. 5 & $6.01 \mathrm{E}-05$ & $1.21 \mathrm{E}-05$ & $1.84 \mathrm{E}-06$ & $4.07 \mathrm{E}-06$ & & $2.88 \mathrm{E}-03$ \\
\hline & Pos. 6 & $2.71 \mathrm{E}-05$ & $2.96 \mathrm{E}-06$ & 4.00E-06 & 4.13E-06 & $8.25 \mathrm{E}-06$ & \\
\hline
\end{tabular}

Table 2. The SAM and SID values calculated for positions 1 to 6 as noted in Figure 3

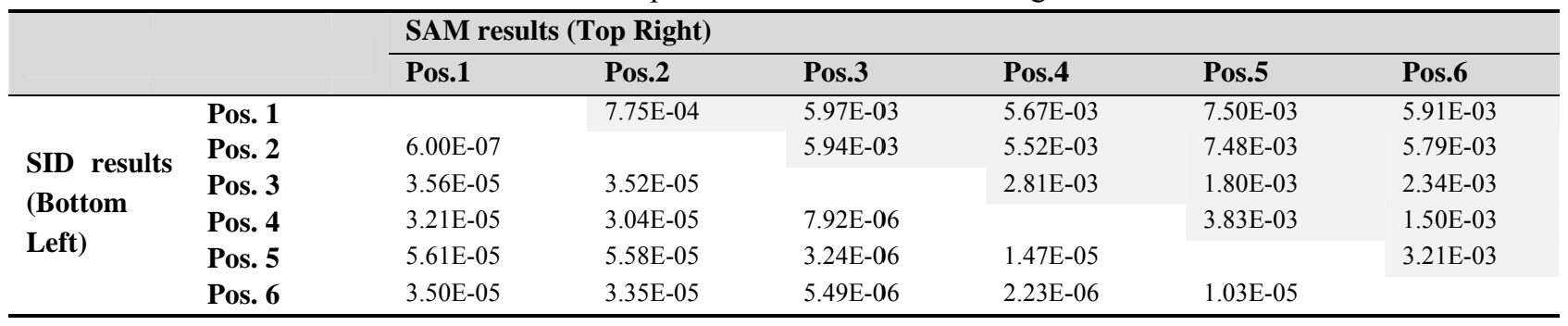


With these tables, it is easy to provide a quantitative measurement of the similarity between selected reference signatures. As indicated in Sections 1.1.1 and 1.1.2, a smaller value signifies that the two positions are more similar. As a result, the background spectra (positions 1 and 2 from Figure 3) are identified as spectrally similar in Table 2, while blood vessel spectra (positions 3-6 from Figures 2 and 3) can also be classified as containing similar substance in Tables 1 and 2. Hence, this justified the correctness of these algorithms while comparing them to the ground truth.

Next, in order to obtain a probabilistic similarity measure between these locations we also calculated the RSDPB-SAM and RSDPB-SID as described in Section 1.1.3. The resulting values for these calculations are tabulated in Tables 3 and 4.

Table 3. The RSDPB-SAM and RSDPB-SID probability based on points 1-6 selected from Figure 2

\begin{tabular}{|c|c|c|c|c|c|c|}
\hline RSDPB-SAM & Pos.1 & Pos.2 & Pos.3 & Pos.4 & Pos.5 & Pos.6 \\
\hline Map 1 & 1 & 0.852 & 0.779 & 0.790 & 0.748 & 0.831 \\
\hline Map 2 & 0.689 & 1 & 0.829 & 0.836 & 0.763 & 0.883 \\
\hline Map 3 & 0.513 & 0.820 & 1 & 0.907 & 0.903 & 0.857 \\
\hline Map 4 & 0.545 & 0.831 & 0.909 & 1 & 0.858 & 0.857 \\
\hline Map 5 & 0.556 & 0.801 & 0.922 & 0.885 & 1 & 0.836 \\
\hline Map 6 & 0.624 & 0.876 & 0.855 & 0.853 & 0.792 & 1 \\
\hline RSDPB-SID & Pos.1 & Pos.2 & Pos.3 & Pos.4 & Pos.5 & Pos.6 \\
\hline Map 1 & 1 & 0.894 & 0.764 & 0.787 & 0.694 & 0.862 \\
\hline Map 2 & 0.565 & 1 & 0.868 & 0.880 & 0.749 & 0.938 \\
\hline Map 3 & 0.230 & 0.895 & 1 & 0.972 & 0.970 & 0.934 \\
\hline Map 4 & 0.272 & 0.900 & 0.971 & 1 & 0.929 & 0.928 \\
\hline Map 5 & 0.304 & 0.860 & 0.979 & 0.953 & 1 & 0.904 \\
\hline Map 6 & 0.416 & 0.936 & 0.914 & 0.911 & 0.822 & 1 \\
\hline
\end{tabular}

Table 4. The RSDPB-SAM and RSDPB-SID probability based on points 1-6 selected from Figure 3

\begin{tabular}{|c|c|c|c|c|c|c|}
\hline RSDPB-SAM & Pos.1 & Pos.2 & Pos.3 & Pos.4 & Pos.5 & Pos.6 \\
\hline Map 1 & 1 & 0.970 & 0.769 & 0.780 & 0.710 & 0.771 \\
\hline Map 2 & 0.970 & 1 & 0.767 & 0.784 & 0.707 & 0.773 \\
\hline Map 3 & 0.683 & 0.685 & 1 & 0.851 & 0.904 & 0.876 \\
\hline Map 4 & 0.707 & 0.715 & 0.855 & 1 & 0.802 & 0.923 \\
\hline Map 5 & 0.685 & 0.686 & 0.924 & 0.839 & 1 & 0.865 \\
\hline Map 6 & 0.685 & 0.691 & 0.875 & 0.920 & 0.829 & 1 \\
\hline RSDPB-SID & Pos.1 & Pos.2 & Pos.3 & Pos.4 & Pos.5 & Pos.6 \\
\hline Map 1 & 1 & 0.996 & 0.777 & 0.798 & 0.648 & 0.781 \\
\hline Map 2 & 0.996 & 1 & 0.774 & 0.804 & 0.641 & 0.784 \\
\hline Map 3 & 0.593 & 0.598 & 1 & 0.909 & 0.963 & 0.937 \\
\hline Map 4 & 0.632 & 0.652 & 0.909 & 1 & 0.832 & 0.974 \\
\hline Map 5 & 0.599 & 0.602 & 0.977 & 0.895 & 1 & 0.926 \\
\hline Map 6 & 0.596 & 0.613 & 0.937 & 0.974 & 0.881 & 1 \\
\hline
\end{tabular}

The results from Tables 3 and 4 further verifies that the background between positions 1 and 2 and the blood vessel positions, 3 to 6 , are similarly very spectrally homogenous. To further demonstrate the selected reference signatures from Figures 2 and 3 and the corresponding spectral similarity within the whole image, the RSDPB-SAM and RSDPB-SID algorithm is further used to process the whole image. Similarity maps for the whole image (see Figures 4 and 5) are generated for the 6 different referencing points. These maps are plotted in pseudocolor to show the likelihood of substantial similarity. 


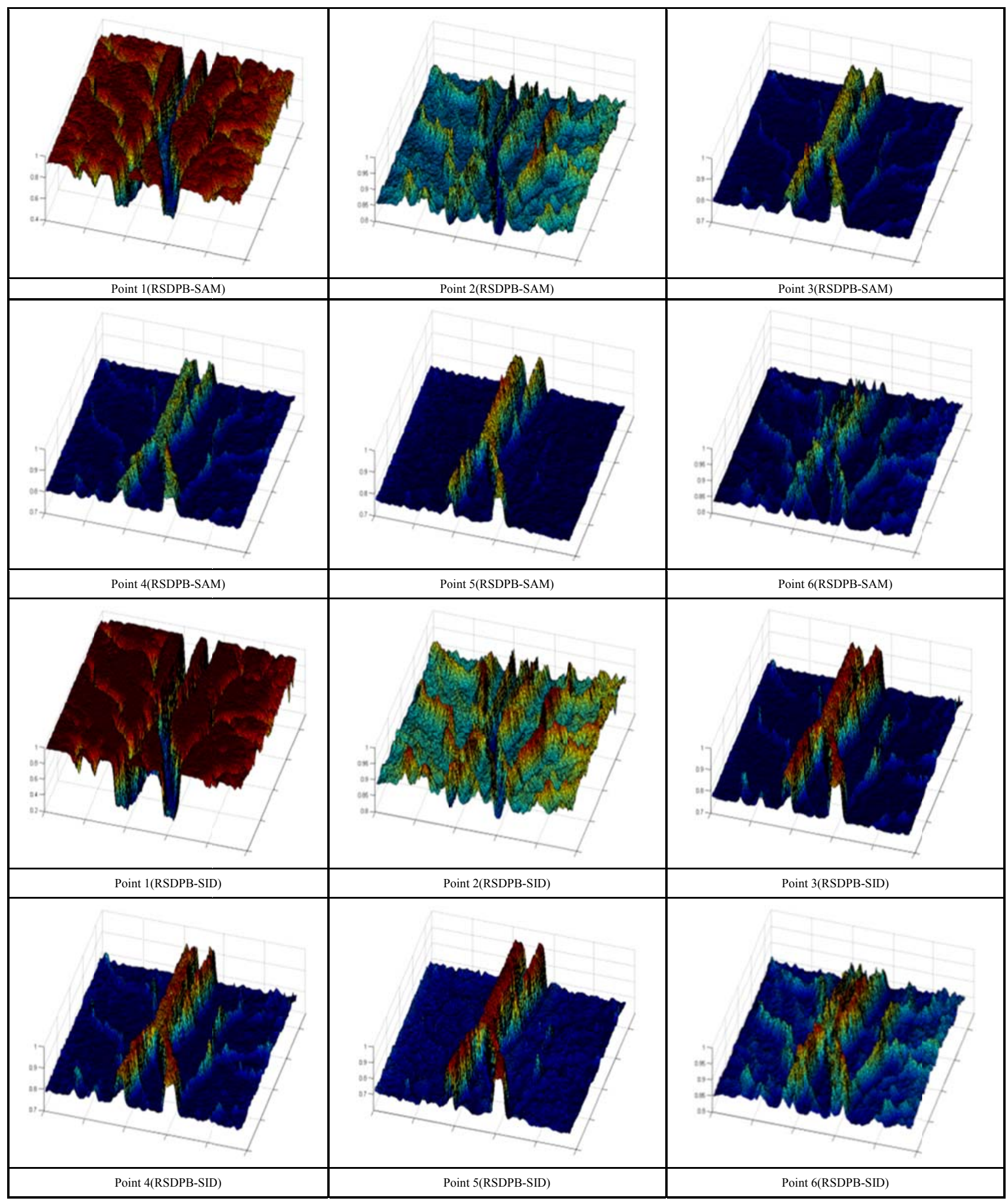

Figure 4. RSDPB (probability of similarity) measurements based on SAM and SID represented on a 3D surface plot. Each point on the surface plot represents the similarity or difference between a selected reference point and the corresponding voxel point from the image in Figure 2. Blue represents no similarity and red represents $100 \%$ similarity between the spectral profiles. 


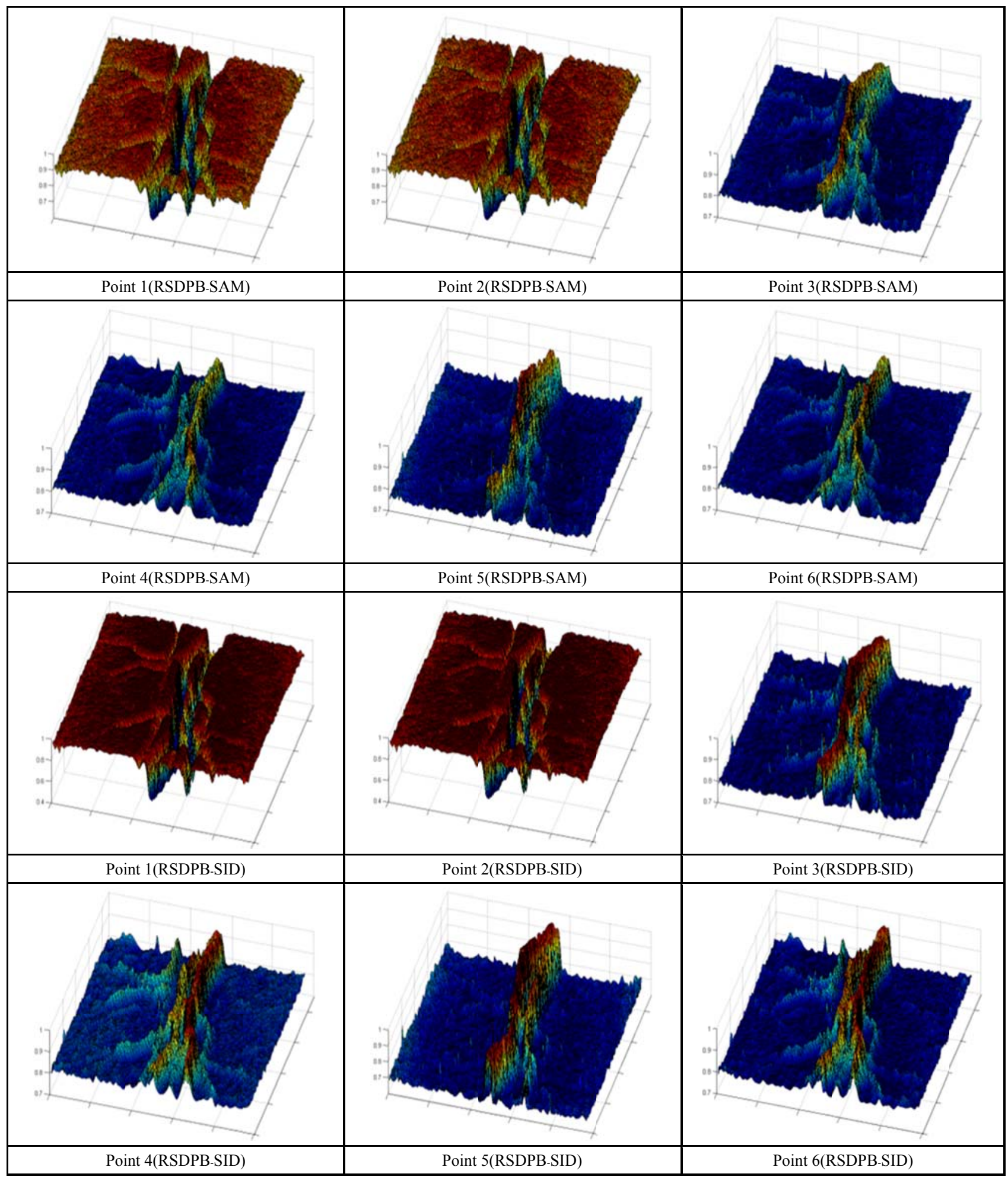

Figure 5. RSDPB (probability of similarity) measurements based on SAM and SID represented on a 3D surface plot. Each point on the surface plot represents the similarity or difference between a selected reference point and the corresponding voxel point from the image in Figure 3. Blue represents no similarity and red represents $100 \%$ similarity between the spectral profiles. 
Both RSDPB-SAM and RSDPB-SID can clearly discriminate the blood vessel from the background tissue where the background tissue has a high similarity (red indicates high probability) to the selected reference point. On the other hand, the blood vessel can be seen to have a lower similarity (blue indicates lower probability). These can be observed in both Figures 4 and 5 in the results for positions 1 and 2. It is worth noting that background reference point 2 in Figure 2 is selected to be a mix between background and blood vessel mixed pixel. As a result, both the blood vessel and background are not showing clear distinction as shown in position 2 in Figure 4. Moreover, the results for blood vessel reference points 3-6 clearly indicates the trail of blood vessels in Figures 4 and 5. It is also worth noting that the reference point selection greatly affects the discriminate analysis. As shown in the results in Figures 4 and 5, the smaller blood vessels are more evident for reference point 4 while not showing for reference point 5 . This might be due to the fact that the spectral signature for reference point 4 is more pure than the signature presented in reference point 5 .

In addition, the RSDPB-SID seems to provide a smoother probabilistic measurement to the referencing points. The resulting images also show small vessels that are hard to identify in the original image shown in Figures 2 and 3. In conclusion, these 3D maps clearly show very good correlation between the anatomic location of large vessels and the high spectral similarity within these vessel pixels. Similarly, there is very good agreement in the spectral classification of background tissue (in this case nerve fiber layer).

\section{Discussions}

We demonstrate that HCTIS can densely sample spectral data from a target scene and produce highly co-registered spectral, spatial, and temporal data from biological specimens in vivo in rabbits. Our data set shows that, by carefully selecting reference spectra in a spectral image, SAM and SID algorithms can identify anatomically similar tissue based only on the respective reflectance spectra. Furthermore, using a RSDPB index, historically used in satellite imagery for classifying similarly structured features, we reliably and reproducibly generate 3D spectral similarity maps of rabbit retinal features in vivo. When reference spectra are set to the voxel locations overlaying retinal vessels and nerve fiber tissue, these similarity maps have excellent agreement with the anatomical patterns of the retinal vessels and nerve fiber tissue, respectively. The resulting agreement between spectral and spatial features in these retinal tissues suggests that the HCTIS discriminatory algorithms are robust across different spectral profiles.

Moreover, the similarity maps demonstrate that the same reference spectra, which reliably and reproducibly identify large retinal vessels (from which the reference spectra are derived), are also able to identify smaller vessels that are branches of the main vessels. This agreement suggests that the spectral discriminatory measures are largely accurate in classifying tissue based on reference spectra. The ability of these discriminatory measures to identify anatomical patterns-and potentially classify normal and pathologic tissues-implies that HCTIS has excellent potential for clinical use as a powerful, non-invasive diagnostic tool.

In the medical field, hyperspectral imaging systems have begun to emerge as a powerful tool for disease diagnosis and image-guided surgery ${ }^{[27]}$. Diagnostically, hyperspectral imaging has demonstrated utility for identifying gastric cancer ${ }^{[28]}$, and in dermatology, for evaluating skin lesions ${ }^{[29]}$. Furthermore, studies in swine show that hyperspectral imaging devices have the potential to become a real-time intraoperative tool for identifying anatomical structures in minimally non-invasive surgeries, such as laparoscopic cholecystectomy, reducing the need for radioactive contrast and aiding surgeons to avoid adverse and serious injury to non-target, vital tissues ${ }^{[30,31]}$.

The utility of hyperspectral imaging systems in measuring oxygen saturation remains an area of ongoing investigation. One study reports optimism about its usefulness for assessing foot perfusion in patients with critical limb ischemia ${ }^{[32]}$. Furthermore, neurosurgeons traditionally obtain postoperative PET scans to assess reperfusion following cerebrovascular reconstruction surgeries; hyperspectral imaging of the cerebral cortex may serve as a promising, real-time, intraoperative 
tool for assessing metabolic changes and successful reperfusion ${ }^{[33]}$. Such real-time imaging could prove a game-changer in assessing the efficacy of surgery and guiding the surgeon intraoperatively.

Hyperspectral systems are emerging with exciting new applications in ophthalmology ${ }^{[34]}$. The majority of these systems integrate with a fundus camera for visualizing the retina, while concurrently providing objective data. Whereas most hyperspectral systems have looked macroscopically at the retina, focusing on its more superficial layers, some investigators have developed a hyperspectral platform integrated with a microscope for analyzing the retina at the cellular level ${ }^{[35]}$. This technique, microscopic pushbroom hyperspectral imaging, which was first developed to examine retinal sections in diabetic and non-diabetic rats ex vivo, represents an exciting imaging modality with the potential to reveal spectral histopathology.

Early on, investigators predicted the utility of hyperspectral imaging systems for detecting early changes in retinal disease when they observed their hyperspectral device's ability to distinguish oxygenated and deoxygenated hemoglobin, and thus, discriminate retinal features such as retinal arteries, veins, and capillary beds ${ }^{[36]}$. Subsequent applications of hyperspectral systems in ophthalmology employed are able to map changes in oxygen saturation of retinal vascular structures, which is important for increasing our understanding of underlying ocular, vascular pathology, from diabetic retinopathy, arteriovenous occlusion, and glaucoma. Early studies in primates showed that a hyperspectral device reliably measured predicted changes in oxygen saturation when breathing experiments and changes to intraocular pressure were induced, suggesting that an oxygen saturation map of the retina can be built ${ }^{[37]}$.

While this system and those used by others ${ }^{[3,4]}$ show clinical promise, these earlier platforms are limited by long acquisition times, often leading to motion artifact and pixel misregistration. Nevertheless, as detailed in Section 1, the introduction of snapshot hyperspectral platforms like the HCTIS enabled significantly faster data acquisition times ${ }^{[14]}$. Most recently, hyperspectral studies in vivo in patients demonstrated significant variations in the retinal vascular oxygen content among normal subjects and patients with diabetic retinopathy; the study also demonstrated for the first time that HCTIS is a clinically feasible method for assessing intravascular oxygen content in humans ${ }^{[38]}$. Previously, the HCTIS oximetry method had been validated in animal models ${ }^{[14,39,40]}$. Snapshot hyperspectral imaging as a tool for evaluating macular pigment and understanding age-related macular degeneration (AMD) is already underway ${ }^{[41,42]}$. Already, its ability to noninvasively evaluate for macular pigment in vivo, appears feasible for macular pigment mapping and AMD evaluation ${ }^{[43]}$.

In conclusion, we show proof-of-principal application of a HCTIS that can densely sample spectral data from a biologically relevant target scene and maintain excellent spatial and temporal coregistration. This hyperspectral data set allows the application of well-tested hyperspectral discriminatory algorithms (SAM, SID and RSDPB) in normal tissue in vivo. The discriminatory analysis shows robust spectral similarity profiles that agree with known anatomical patterns. The ease and safety of the HCTIS imaging modality and the robust discriminatory algorithms may be a clinically useful tool for non-invasive analysis of normal and pathologic tissue in the future.

Future challenges include determining the optimal reference spectra and in creating a library of reference spectra for important biological markers. Already, we have begun to identify spectral signatures for oxygenated and deoxygenated hemoglobin and macular pigment components like lutein and zeaxanthin ${ }^{[42]}$. Oncologic biomarkers may provide the next logical progression for this exciting technology. With the establishment of optimal reference spectra and improvement in acquisition and computing time, there will be countless ocular and medical diseases that will benefit from the objective data that this imaging modality provides.

\section{Acknowledgements}

This work was supported by the National Science Foundation Engineering Research Center for Biomimetic Microelectronic Devices (NSF EEC-0310723), by Reichert Technologies, Inc., and by an unrestricted grant from Research to 
Prevent Blindness, New York, NY 10022. A. H. Kashani and M. S. Humayun have intellectual property related to the subject matter of this article. G. Martin is an employee of Reichert Technologies, Inc., which has a commercial interest in the subject matter of this article. The other authors have no financial/conflicting interests to disclose.

\section{References}

[1] Rafert B, Holbert E, Sellar RG, et al. Hyperspectral observations of space objects. Proc. SPIE 2198, Instrumentation in Astronomy VIII. 1994 Jun; 2198: 1414-24. http://dx.doi.org/10.1117/12.176727

[2] Harris A, Dinn RB, Kagemann L, et al. A review of methods for human retinal oximetry. Ophthalmic Surg Lasers Imaging. 2003 Mar-Apr; 34(2): 152-64. PMid:12665234.

[3] Mordant DJ, Al-Abboud I, Muyo G, et al. Spectral imaging of the retina. Eye (Lond). 2011 Mar; (3): 309-20. PMid:21390065. http://dx.doi.org/10.1038/eye.2010.222

[4] Mordant DJ, Al-Abboud I, Muyo G, et al. Validation of human whole blood oximetry, using a hyperspectral fundus camera with a model eye. Invest Ophthalmol Vis Sci. 2011 Apr; 52(5): 2851-9. PMid:21220553. http://dx.doi.org/10.1167/iovs.10-6217

[5] Sorg BS, Moeller BJ, Donovan O, et al. Hyperspectral imaging of hemoglobin saturation in tumor microvasculature and tumor hypoxia development. J Biomed Opt. 2005 Jul-Aug; 10(4): 44004. PMid:16178638. http://dx.doi.org/10.1117/1.2003369

[6] Martin ME, Wabuyele MB, Chen K, et al. Development of an advanced hyperspectral imaging (HSI) system with applications for cancer detection. Ann Biomed Eng. 2006 Jun; 34(6): 1061-8. PMid:16783661. http://dx.doi.org/10.1007/s10439-006-9121-9

[7] Schultz RA, Nielsen T, Zavaleta JR, et al. Hyperspectral imaging: a novel approach for microscopic analysis. Cytometry. 2001 Apr; 43(4): 239-47. http://dx.doi.org/10.1002/1097-0320(20010401)43:4<239::AID-CYTO1056>3.0.CO;2-Z

[8] Timlin JA, Haaland DM, Sinclair MB, et al. Hyperspectral microarray scanning: impact on the accuracy and reliability of gene expression data. BMC Genomics. 2005 May; 6: 72. PMid:15888208. http://dx.doi.org/10.1186/1471-2164-6-72

[9] Pittman RN, Duling BR. A new method for the measurement of percent oxyhemoglobin. J Appl Physiol. 1975 Feb; $38(2): 315-20$. PMid:1120757.

[10] Pittman RN, Duling BR. Measurement of percent oxyhemoglobin in the microvasculature. J Appl Physiol. 1975 Feb; $38(2): 321-7$. PMid:1120758

[11] Liang H. Advances in multispectral and hyperspectral imaging for archaeology and art conservation. Applied Physics A. 2012: 106(2): 309-23. http://dx.doi.org/10.1007/s00339-011-6689-1

[12] Edelman G, van Leeuwen TG, Aalders MC. Hyperspectral imaging for the age estimation of blood stains at the crime scene. Forensic Sci Int. 2012 Nov 30; 223(1-3): 72-7. PMid:22938693. http://dx.doi.org/10.1016/j.forsciint.2012.08.003

[13] Kuula J, Pölönen I, Puupponen H, et al. Using VIS/NIR and IR spectral cameras for detecting and separating crime scene details. Proc. SPIE, Sensors, and Command, Control, Communications, and Intelligence (C3I) Technologies for Homeland Security and Homeland Defense XI, 83590P. 2012 May: 8359.

[14] Johnson WR, Wilson DW, Fink W, et al. Snapshot hyperspectral imaging in ophthalmology. J Biomed Opt. 2007 Jan-Feb; 12(1): 014036. PMid:17343511. http://dx.doi.org/10.1117/1.2434950

[15] Delori FC. Noninvasive technique for oximetry of blood in retinal vessels. Appl Opt. 1988 Mar; 27(6): 1113-25. PMid:20531526. http://dx.doi.org/10.1364/AO.27.001113

[16] Descour MR, Dereniak EL. Nonscanning, no-moving parts imaging spectrometer. Proc SPIE. 1995 Jun; $2480: 48-64$. http://dx.doi.org/10.1117/12.210908

[17] Descour M, Dereniak E. Computed-tomography imaging spectrometer: experimental calibration and reconstruction results. Appl Opt. 1995 Aug; 34(22): 4817-26. PMid:21052321. http://dx.doi.org/10.1364/AO.34.004817

[18] Chang CI. An information-theoretic approach to spectral variability, similarity, and discrimination for hyperspectral image analysis. Information Theory, IEEE Transactions on. 2000 Aug; 46(5): 1927-32.

[19] Dennison PE, Halligan KQ, Roberts DA. A comparison of error metrics and constraints for multiple endmember spectral mixture analysis and spectral angle mapper. Remote Sensing of Environment. 2004 November; 93(3): 359-67. http://dx.doi.org/10.1016/j.rse.2004.07.013

[20] Robila SA, Gershman A. Spectral matching accuracy in processing hyperspectral data. International Symposium on Signals, Circuits and Systems. 2005 July: 163-6. http://dx.doi.org/10.1109/isscs.2005.1509878

[21] Yang C, Everitt JH, Bradford JM. Yield estimation from hyperspectral imagery using spectral angle mapper (SAM). Transactions of the ASAE. 2008; 51(2): 729-37. http://dx.doi.org/10.13031/2013.24370

[22] Hecker C, van der Meijde M, van der Werff H, et al. Assessing the Influence of Reference Spectra on Synthetic SAM Classification Results. Geoscience and Remote Sensing. IEEE Transactions on. 2008 Dec; 46(12): 4162-72. 
[23] Chang CI. An information-theoretic approach to spectral variability, similarity, and discrimination for hyperspectral image analysis. Information Theory, IEEE Transactions on. 2000 Aug; 46(5): 1927-32.

[24] Du Y, Chang CI, Ren H, et al. New hyperspectral discrimination measure for spectral characterization. Optical Engineering. 2004; 43: 1777-84. http://dx.doi.org/10.1117/1.1766301

[25] Johnson WR, Wilson DW, Fink W, et al. Snapshot hyperspectral imaging in ophthalmology. J Biomed Opt. 2007 Jan-Feb; 12(1): 014036. PMid:17343511. http://dx.doi.org/10.1117/1.2434950

[26] Cho MA, Mathieu R, Debba P. Multiple endmember spectral-angle-mapper (sam) analysis improves discrimination of savanna tree species, in Hyperspectral Image and Signal Processing: Evolution in Remote Sensing, 2009. WHISPERS '09. First Workshop on. 2009 Aug: 1-4.

[27] Lu G, Fei B. Medical hyperspectral imaging: a review. J Biomed Opt. 2014 Jan; 19(1): 10901. PMid:24441941. http://dx.doi.org/10.1117/1.JBO.19.1.010901

[28] Goto A, Nishikawa J, Kiyotoki S, et al. Use of hyperspectral imaging technology to develop a diagnostic support system for gastric cancer. J Biomed Opt. 2015 Jan; 20(1): 016017. PMid:25604546. http://dx.doi.org/10.1117/1.JBO.20.1.016017

[29] Koprowski R, Wilczyński S, Wróbel Z, et al. Calibration and segmentation of skin areas in hyperspectral imaging for the needs of dermatology. Biomed Eng Online. 2014 Aug 8; 13: 113. PMid:25103039. http://dx.doi.org/10.1186/1475-925X-13-113

[30] Zuzak KJ, Naik SC, Alexandrakis G, et al. Characterization of a near-infrared laparoscopic hyperspectral imaging system for minimally invasive surgery. Anal Chem. 2007 Jun 15; 79(12): 4709-15. PMid:17492839. http://dx.doi.org/10.1021/ac070367n

[31] Zuzak KJ, Naik SC, Alexandrakis G, et al. Intraoperative bile duct visualization using near-infrared hyperspectral video imaging. Am J Surg. 2008 Apr; 195(4): 491-7. PMid:18304512. http://dx.doi.org/10.1016/j.amjsurg.2007.05.044

[32] Benitez E, Sumpio BJ, Chin J, et al. Contemporary assessment of foot perfusion in patients with critical limb ischemia. Semin Vasc Surg. 2014 Mar; 27(1): 3-15. PMid:25812754. http://dx.doi.org/10.1053/j.semvascsurg.2014.12.001

[33] Mori M, Chiba T, Nakamizo A, et al. Intraoperative visualization of cerebral oxygenation using hyperspectral image data: a two-dimensional mapping method. Int J Comput Assist Radiol Surg. 2014 Apr 16 [Epub ahead of print]. PMid:24737109. http://dx.doi.org/10.1007/s11548-014-0989-9

[34] Clarkson D. Hyperspectral imaging in ophthalmology. The Optician. 2015 Feb 13; 249: 24-6.

[35] Li Q, Xue Y, Xiao G, et al. New microscopic pushbroom hyperspectral imaging system for application in diabetic retinopathy research. J Biomed Opt. 2007 Nov-Dec; 12(6): 064011. PMid:18163827. http://dx.doi.org/10.1117/1.2821210

[36] Harvey AR, Lawlor J, McNaught AI, et al. Hyperspectral imaging for the detection of retinal disease. Proc. SPIE. 2002 Nov; 4816 : 325-35. http://dx.doi.org/10.1117/12.451693

[37] Khoobehi B, Beach JM, Kawano H. Hyperspectral imaging for measurement of oxygen saturation in the optic nerve head. Invest Ophthalmol Vis Sci. 2004 May; 45(5): 1464-72. PMid:15111603. http://dx.doi.org/10.1167/iovs.03-1069

[38] Kashani AH, Lopez Jaime GR, Saati S, et al. Noninvasive assessment of retinal vascular oxygen content among normal and diabetic human subjects: a study using hyperspectral computed tomographic imaging spectroscopy. Retina. 2014 Sep; 34(9): 1854-60. PMid:24732694. http://dx.doi.org/10.1097/IAE.0000000000000146

[39] Jaime GR, Kashani AH, Saati S, et al. Acute variations in retinal vascular oxygen content in a rabbit model of retinal venous occlusion. PLoS One. 2012; 7(11): e50179. PMid:23185567. http://dx.doi.org/10.1371/journal.pone.0050179

[40] Kashani AH, Kirkman E, Martin G, et al. Hyperspectral computed tomographic imaging spectroscopy of vascular oxygen gradients in the rabbit retina in vivo. PLoS One. 2011; 6(9): e24482. PMid:21931729. http://dx.doi.org/10.1371/journal.pone.0024482

[41] Sohrab MA, Smith RT, Fawzi AA. Imaging characteristics of dry age-related macular degeneration. Semin Ophthalmol. 2011 May; 26(3): 156-66. PMid:21609229. http://dx.doi.org/10.3109/08820538.2011.570848

[42] Lee N, Wielaard J, Fawzi AA, et al. In vivo snapshot hyperspectral image analysis of age-related macular degeneration. Conf Proc IEEE Eng Med Biol Soc. 2010; 2010: 5363-6. http://dx.doi.org/10.1109/iembs.2010.5626463

[43] Fawzi AA, Lee N, Acton JH, et al. Recovery of macular pigment spectrum in vivo using hyperspectral image analysis. J Biomed Opt. 2011 Oct; 16(10): 106008. PMid:22029355. http://dx.doi.org/10.1117/1.3640813 